\title{
Adaptation to Noise in Human Speech Recognition Depends on Noise-Level Statistics and Fast Dynamic-Range Compression
}

\author{
Miriam I. Marrufo-Pérez, ${ }^{1,2}$ Dora del Pilar Sturla-Carreto, ${ }^{1}$ Almudena Eustaquio-Martín, ${ }^{1,2}$ and \\ (D) Enrique A. Lopez-Poveda ${ }^{1,2,3}$ \\ ${ }^{1}$ Instituto de Neurociencias de Castilla y León, Universidad de Salamanca, 37007 Salamanca, Spain, ${ }^{2}$ Instituto de Investigación Biomédica, \\ Universidad de Salamanca, 37007 Salamanca, Spain, and ${ }^{3}$ Departamento de Cirugía, Facultad de Medicina, Universidad de Salamanca, 37007 \\ Salamanca, Spain
}

Human hearing adapts to background noise, as evidenced by the fact that listeners recognize more isolated words when words are presented later rather than earlier in noise. This adaptation likely occurs because the leading noise shifts ("adapts") the dynamic range of auditory neurons, which can improve the neural encoding of speech spectral and temporal cues. Because neural dynamic range adaptation depends on stimulus-level statistics, here we investigated the importance of "statistical" adaptation for improving speech recognition in noisy backgrounds. We compared the recognition of noised-masked words in the presence and in the absence of adapting noise precursors whose level was either constant or was changing every $50 \mathrm{~ms}$ according to different statistical distributions. Adaptation was measured for 28 listeners ( 9 men) and was quantified as the recognition improvement in the precursor relative to the no-precursor condition. Adaptation was largest for constantlevel precursors and did not occur for highly fluctuating precursors, even when the two types of precursors had the same mean level and both activated the medial olivocochlear reflex. Instantaneous amplitude compression of the highly fluctuating precursor produced as much adaptation as the constant-level precursor did without compression. Together, results suggest that noise adaptation in speech recognition is probably mediated by neural dynamic range adaptation to the most frequent sound level. Further, they suggest that auditory peripheral compression per se, rather than the medial olivocochlear reflex, could facilitate noise adaptation by reducing the level fluctuations in the noise.

Key words: auditory compression; dynamic range adaptation; medial olivocochlear reflex; speech recognition; statistical adaptation

\section{Significance Statement}

Recognizing speech in noise is challenging but can be facilitated by noise adaptation. The neural mechanisms underlying this adaptation remain unclear. Here, we report some benefits of adaptation for word-in-noise recognition and show that (1) adaptation occurs for stationary but not for highly fluctuating precursors with equal mean level; (2) both stationary and highly fluctuating noises activate the medial olivocochlear reflex; and (3) adaptation occurs even for highly fluctuating precursors when the stimuli are passed through a fast amplitude compressor. These findings suggest that noise adaptation reflects neural dynamic range adaptation to the most frequent noise level and that auditory peripheral compression, rather than the medial olivocochlear reflex, could facilitate noise adaptation.

Received Feb. 27, 2020; revised May 3, 2020; accepted July 6, 2020

Author contributions: M.I.M.-P. and D.d.P.S.-C. performed research; M.I.M.-P. analyzed data; M.I.M.-P. and D.d.P.S.-C. wrote the first draft of the paper; M.I.M.-P. and E.A.L.-P. edited the paper; M.I.M.-P. and E.A.L.-P. wrote the paper; A.E.-M. contributed unpublished reagents/analytic tools; E.A.L.-P. designed research.

The authors declare no competing financial interests.

This work was supported by University of Salamanca and Banco Santander doctoral contract to M.I.M.-P., European Regional Development Fund, and Spanish Ministry of Economy and Competitiveness BFU201565376-P to E.A.L.-P. We thank Peter T. Johannesen for help with otoacoustic-emission data collection.

Correspondence should be addressed to Enrique A. Lopez-Poveda at ealopezpoveda@usal.es.

https://doi.org/10.1523/JNEUROSCI.0469-20.2020

Copyright $\odot 2020$ the authors

\section{Introduction}

Accurate speech recognition is necessary for human communication and socialization (Higgins and Semin, 2001) but can be challenging in noisy environments. Normal-hearing listeners presented with speech in noise, however, adapt out of the simultaneous noise and can recognize up to $28 \%$ more isolated words or syllables when the speech tokens are presented in continuous noise than when the tokens start at the same time as the noise (Ainsworth and Meyer, 1994; Cervera and Gonzalez-Alvarez, 2007). Noise adaptation likely occurs because the leading noise 
adapts the dynamic range of auditory neurons depending on the noise level (Ainsworth and Meyer, 1994), but the physiological processes underlying noise adaptation remain unclear.

Like visual (Ohzawa et al., 1982) or somatosensory neurons (Garcia-Lazaro et al., 2007), auditory neurons adapt their response dynamic range to the current environment. For short tones in continuous, steady noise, neurons shift their discharge rate-level functions toward the noise level (Evans, 1974; Costalupes et al., 1984; Gibson et al., 1985; Phillips and Hall, 1986; Rees and Palmer, 1989). In steady noise, neural dynamic range adaptation (DRA) toward the mean noise level can increase the accuracy with which auditory neurons encode sound intensity, which might improve the encoding of the speech envelope (Fig. 1A) (Marrufo-Pérez et al., 2018) and/or the speech spectrum as represented in the auditory nerve (Fig. 1B) (Ainsworth and Meyer, 1994).

Auditory neurons, however, do not simply adapt their dynamic range to the mean sound level but to the most probable levels in the stimulus (Dean et al., 2005). Indeed, when the stimulus (noise or tone) level varies every $50 \mathrm{~ms}$ according to a statistical distribution of sound levels, and a set of levels is presented $80 \%$ of the time (defined as "high probability region"), neurons shift their rate level functions toward the "most commonly occurring sound levels" (Dean et al., 2005, 2008; Watkins and Barbour, 2008, 2011; Wen et al., 2009, 2012). When the stimulus level is distributed in two high-probability regions, neurons do not adapt to the mean level (which lays between the two regions). Instead, some neurons shift their dynamic range toward one level region and other neurons shift it toward the other region (Dean et al., 2005). These adjustments improve the coding precision of levels near the high probability region in the neural population (Dean et al., 2005; Wen et al., 2009; but see Rocchi and Ramachandran, 2018). Most auditory neurons adapt with a time course of 100-400 ms (Dean et al., 2008; Wen et al., 2012), and DRA has been observed in the auditory nerve (Wen et al., 2009, 2012), the inferior colliculus (Dean et al., 2005, 2008; Robinson et al., 2016; Bakay et al., 2018), and the auditory cortex (Watkins and Barbour, 2008, 2011) of animals, as well as in the human auditory cortex (Herrmann et al., 2018, 2019).

The aim of the present study was to investigate how noise adaptation in human speech recognition depends on the noise level distribution. We compared adaptation for noise-masked words preceded by adapting noise precursors with different noise level distributions, going from steady, to mildly fluctuating to highly fluctuating. Neural DRA was expected to be less for the highly fluctuating precursor because it does not provide a "most common" level to which neurons can adapt their dynamic ranges. We also investigated the effects of fast-amplitude compression on noise adaptation. Fast-amplitude compression could facilitate adaptation by turning a highly fluctuating sound into a less fluctuating one, thus allowing auditory neurons to adapt to a prevailing level. Fast compression occurs at multiple auditory processing stages, is often reduced in impaired hearing, and is applied by many hearing devices (see Bacon et al., 2004). If it facilitates adaptation, this might shed light on why speech-in-noise recognition is more challenging for hearing-impaired than for normal-hearing listeners, even in aided conditions (e.g., Glasberg and Moore, 1989; Johannesen et al., 2016).

\section{Material and Methods}

\section{Experimental design}

We compared the signal-to-noise ratio (SNR) at 50\% word recognition (termed the speech reception threshold [SRT]) for
A
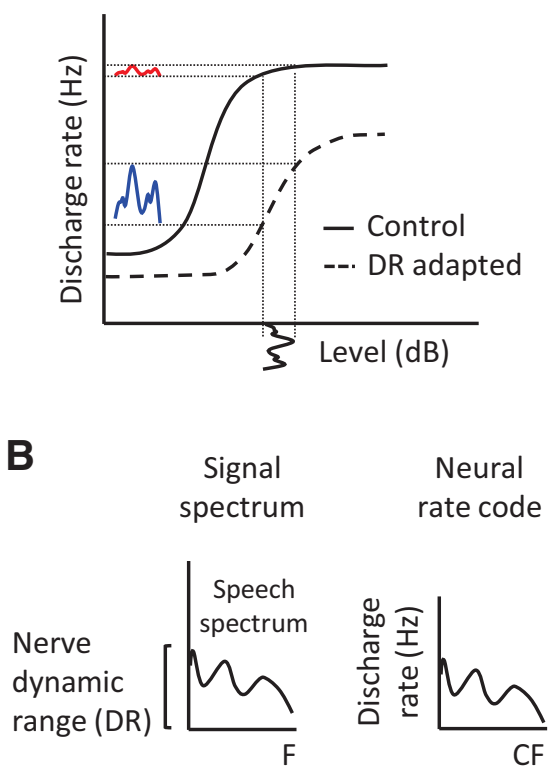

WithoutDRA
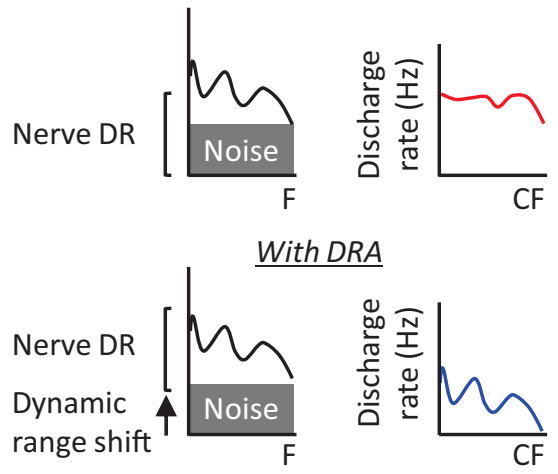

Figure 1. Effects of DRA on the encoding of the speech envelope $(\boldsymbol{A})$ and the speech spectrum $(\boldsymbol{B}) . \boldsymbol{A}$, The shift of the neuronal dynamic range toward the noise level can enhance the coding of the speech envelope. A given envelope (black trace next to the abscissa) would be better represented in the discharge rate of the adapted auditory neuron (blue envelope) than in the discharge rate of the nonadapted neuron (red envelope). Figure adapted with permission from Marrufo-Pérez et al. (2018). B, Top, In quiet, the speech spectrum is faithfully encoded in the auditory nerve rate profile. For speech in noise, however, many auditory nerve fibers would be saturated at high intensities because of their limited dynamic range. This would result in a degradation of the speech spectrum as coded in the auditory nerve rate profile (red representation). Conversely, the speech spectrum would be more faithfully encoded by the noise-adapted neurons because their responses would saturate at higher intensities (blue). F, Acoustic frequency; $(F$, auditory nerve characteristic frequency. Figure adapted with permission from Ainsworth and Meyer (1994).

noise-masked words in the presence and in the absence of an adapting noise precursor (1.5 $\mathrm{s}$ in duration). The long-term spectra of the (simultaneous) masking noise and the precursor noise were identical and equal to the long-term spectrum of speech (speech-shaped noise [SSN]). The SRT improvement in the precursor relative to the no precursor condition was regarded as the amount of adaptation to noise.

To investigate the effect of precursor-level statistics on noise adaptation, SRTs were measured without the adapting precursor and with three precursor types: steady (with a constant level of $65 \mathrm{~dB}$ SPL), mildly fluctuating (the precursor level changed every $50 \mathrm{~ms}$ and $80 \%$ of the $50 \mathrm{~ms}$ segments were at $65 \mathrm{~dB} \mathrm{SPL}$ ), or highly fluctuating (with only $20 \%$ of the $50 \mathrm{~ms}$ segments at $65 \mathrm{~dB}$ $\mathrm{SPL})$. We hypothesized that the less fluctuating the precursor 
level is, the greater the amount of adaptation to noise. The change in precursor-level statistics resulted in the mildly and highly fluctuating precursors having mean levels 1.5 and $3.7 \mathrm{~dB}$ higher than the level of the steady precursor, respectively. We investigated how this difference in mean level affected noise adaptation by measuring adaptation for a steady precursor with a mean level equal to that of the highly fluctuating precursor.

To investigate the effects of amplitude compression on noise adaptation, we measured SRTs without a precursor as well as with a highly fluctuating precursor, and stimuli were passed through a broadband, instantaneous amplitude compressor before presenting them to the listeners. This compression was expected to distort the speech tokens and thus to worsen SRTs overall, but it was also expected to facilitate (even reinstate) noise adaptation by reducing the level fluctuations in the adapting precursor noise. Measurements were obtained for two different compression ratios $(2: 1$ and $4: 1 \mathrm{~dB} / \mathrm{dB})$.

Speech signals are filtered by the cochlea and decomposed into several bandpass-filtered signals, each of which can be separated in an envelope (that represents the slow amplitude fluctuations over time) and the temporal fine structure (the rapid fluctuations with a rate close to the center frequency of the bandpass filter) (Rosen, 1992). Noise adaptation in word recognition is more obvious for words processed ("vocoded") to maintain only the envelope cues in speech than for natural (unprocessed) words (Marrufo-Pérez et al., 2018). For this reason and to better reveal the hypothesized effects of noise level statistics and compression on noise adaptation, SRTs were measured for vocoded as well as natural (unprocessed) words.

The processes responsible for neuronal DRA toward the sound-level statistics are unclear, but DRA might be mediated to some uncertain extent by the medial olivocochlear reflex (MOCR) (Dean et al., 2005, 2008), an efferent pathway that projects from the auditory midbrain to the cochlea (for review, see Guinan, 2006; Lopez-Poveda, 2018). A control experiment was conducted to investigate the potential involvement of the MOCR on noise adaptation. MOCR activation hyperpolarizes outer hair cells (OHCs) (Cooper and Guinan, 2003), which linearizes cochlear mechanical responses by turning down the gain of the cochlear amplifier for low-to-mid level sounds at or near the characteristic frequency (Murugasu and Russell, 1996; Dolan et al., 1997; Cooper and Guinan, 2003, 2006; Guinan, 2006). This is expected to reduce the level of otoacoustic emissions, a byproduct of a nonlinear cochlea (Collet et al., 1990; Berlin et al., 1993; Guinan, 2006). Here, we measured the change in the level of click evoked otoacoustic emissions (CEOAEs) for steady and highly fluctuating contralateral noises and compared the pattern of this change with the pattern of noise adaptation in the word recognition tests. Mertes (2018) showed that contralateral broadband steady noise produced statistically similar suppression of CEOAEs as did speech-modulated noise. Furthermore, ipsilateral broadband noise is as effective as contralateral broadband noise in activating the MOCR (Lilaonitkul and Guinan, 2009). Therefore, if the contralateral steady and highly fluctuating noises suppressed CEOAEs equally but adaptation in speech recognition occurred only for the steady noise, this would undermine the notion that noise adaptation is mediated by the MOCR.

Methods were approved by the Ethics Committee of the University of Salamanca (Spain).

\section{Noise adaptation in speech recognition}

Participants. Twenty-eight human listeners (9 men) with normal hearing $(\mathrm{NH})$ participated in the main experiment (mean \pm
SD age $=26.6 \pm 8.3$ years). Twenty-five of them had audiometric thresholds $\leq 20 \mathrm{~dB}$ hearing level (HL) in the test (left) ear at octave frequencies between $125 \mathrm{~Hz}$ and $8 \mathrm{kHz}$ (American National Standards Institute, 1996). Three participants had audiometric thresholds equal to $25 \mathrm{~dB} \mathrm{HL}$ at one or two audiometric frequencies. Fourteen listeners were tested with natural and vocoded words, 13 listeners were tested with only natural words, and 1 listener was tested with only vocoded words. In total, 27 and 15 participants were tested with natural and vocoded words, respectively.

All participants were native speakers of Spanish. They were volunteers and not paid for their services. All of them signed an informed consent to participate in the study.

Stimuli. Stimuli were presented monaurally to the left ear of the participants. Thirty-five disyllabic words were used to measure each SRT. The first 10 words were always the same but were presented in random order. They were used to give participants the opportunity to become familiar with the test condition. The last 25 words corresponded to one of the nine phonetically balanced lists from Cárdenas and Marrero (1994). Test words were presented in random order across test conditions to minimize the possibility that participants remembered the words.

The vocoder included a high-pass pre-emphasis filter (firstorder Butterworth filter with a $3 \mathrm{~dB}$ cutoff frequency of $1.2 \mathrm{kHz}$ ); a bank of 12, sixth-order Butterworth bandpass filters whose 3 $\mathrm{dB}$ cutoff frequencies followed a modified logarithmic distribution between 100 and $8500 \mathrm{~Hz}$; and envelope extraction via full-wave rectification and low-pass filtering (fourth-order Butterworth low-pass filter with a $3 \mathrm{~dB}$ cutoff frequency of $400 \mathrm{~Hz}$ ). The envelope for each frequency channel was used to modulate the amplitude of a sinusoidal carrier at the channel center frequency, and the modulated signals were sample-wise added to obtain the vocoded speech.

In the no-precursor condition, the simultaneous noise masker started $50 \mathrm{~ms}$ before the word onset and finished $50 \mathrm{~ms}$ after the word offset (including $10 \mathrm{~ms}$ onset and offset ramps) (Fig. 2A). In the precursor conditions, the noise precursor started $1500 \mathrm{~ms}$ before the word onset. The simultaneous masking noise was steady, and its level was fixed at $65 \mathrm{~dB}$ SPL. By contrast, the level of the precursor noise varied every $50 \mathrm{~ms}$ depending on the precursor type. For the steady precursor, the level was constant at $65 \mathrm{~dB}$ SPL (Fig. 2B). For the mildly fluctuating precursor, the level of $80 \%$ of the $50 \mathrm{~ms}$ segments was at $65 \mathrm{~dB}$ SPL and the level of the remaining $20 \%$ of the segments varied uniformly between 20 and $80 \mathrm{~dB}$ SPL in $5 \mathrm{~dB}$ steps (Fig. 2C). For the highly fluctuating precursor, the level of $20 \%$ of the $50 \mathrm{~ms}$ segments was at $65 \mathrm{~dB}$ SPL and the level of the remaining $80 \%$ of the segments varied uniformly between 20 and $80 \mathrm{~dB}$ SPL in $5 \mathrm{~dB}$ steps (Fig. $2 D)$. The different sound levels were presented randomly throughout the duration of the precursor. To generate the fluctuating precursors, a steady SSN of $65 \mathrm{~dB}$ SPL was multiplied by the desired envelope, whose amplitude varied in $50 \mathrm{~ms}$ segments. Different masking and precursor noises were generated for each word (i.e., noises were not "frozen").

The level of the steady precursor was $65 \mathrm{~dB}$ SPL, thus equal to the level of the simultaneous masking noise, but the change in precursor-level statistics resulted in the mildly and highly fluctuating precursors having mean levels of $66.5 \mathrm{~dB}$ SPL ( $\pm 1.9 \mathrm{SD}$ ) and $68.7 \mathrm{~dB}$ SPL $( \pm 0.8 \mathrm{SD})$, respectively, thus different from the level of the simultaneous masking noise. A complementary experiment was performed to investigate whether this difference in the mean level may affect the amount of noise adaptation (see below). 

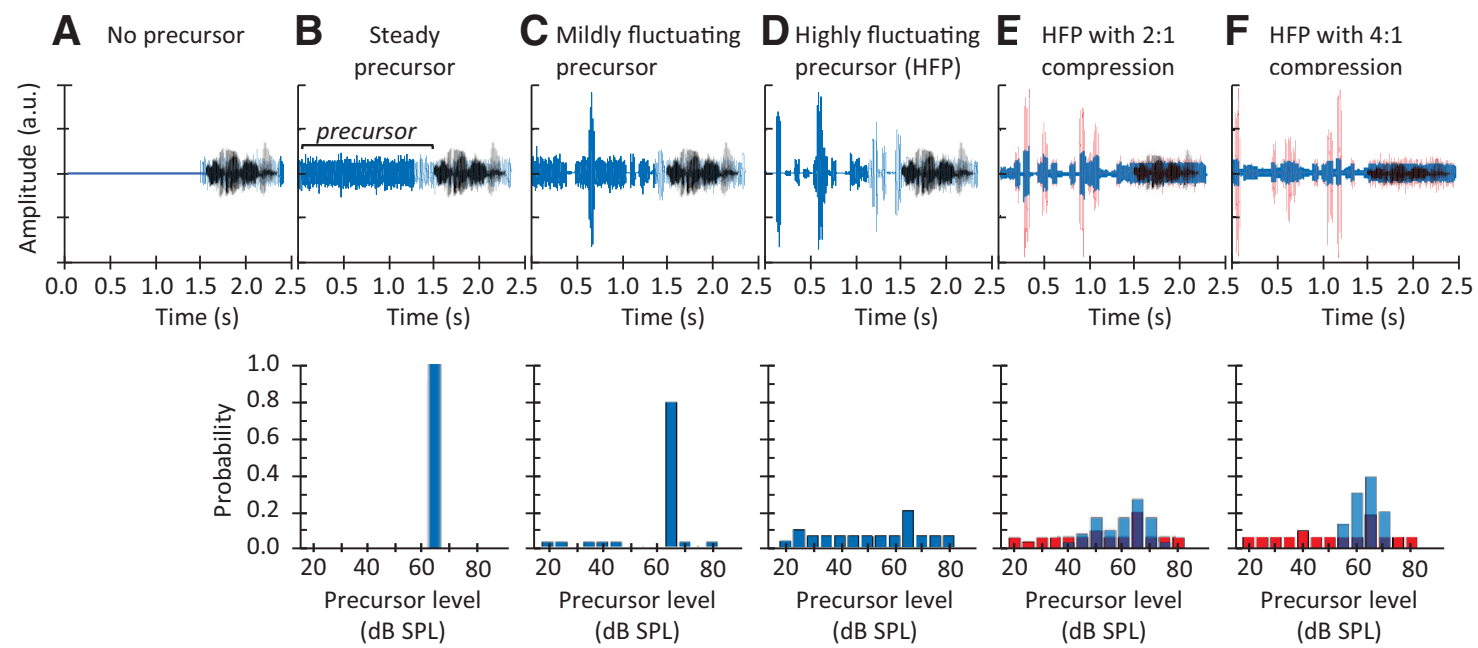

Figure 2. Example stimuli used to measure the effect of precursor-level statistics on noise adaption. Top, Stimulus waveforms for conditions with no precursor $(\boldsymbol{A})$, steady precursor $(\boldsymbol{B})$, mildly fluctuating precursor $(\boldsymbol{C})$, and highly fluctuating precursor (HFP) $(\boldsymbol{D})$, and compressed highly fluctuating precursor with a compression ratio 2:1 (E) or 4:1 $(\boldsymbol{F})$. $(-\boldsymbol{F}$, The precursor level fluctuated every $50 \mathrm{~ms}$, but words (depicted in black for illustration purposes) were masked by simultaneous steady noise. Bottom, Precursor-level distributions. The probability was calculated by considering each $50 \mathrm{~ms}$ segment as 1 unit. $\boldsymbol{B}-\boldsymbol{F}$, Precursor level distributions before (red) and after (blue) applying amplitude compression. a.u., arbitrary unit.

The effects of instantaneous amplitude compression on noise adaptation were assessed by measuring SRTs without and with a highly fluctuating precursor but passing stimuli through a broadband, instantaneous amplitude compressor before presenting them to the listeners. Two compression ratios were used (2:1 and $4: 1 \mathrm{~dB} / \mathrm{dB})$. The instantaneous amplitude of the compressed stimulus, $y[i]$, was calculated as follows:

$$
y[i]=\operatorname{sign}(x[i]) \cdot b \cdot|x[i]|^{c}
$$

where $i$ denotes sample number, $c$ is a parameter that determines the amount of compression (i.e., 0.25 and 0.5 for compression ratios $4: 1$ and 2:1, respectively), $x[i]$ is the instantaneous amplitude of the uncompressed stimulus, and $b$ was set so that an input level of $65 \mathrm{~dB}$ SPL corresponded to an output level of $65 \mathrm{~dB}$ SPL. This resulted in sound levels $<65 \mathrm{~dB}$ SPL being amplified, levels equal to $65 \mathrm{~dB}$ SPL not changing, and levels $>65 \mathrm{~dB}$ SPL being attenuated (Fig. 3). Figure 2E,F shows example compressed stimuli.

Procedure. To measure an SRT, the speech level varied adaptively using a one-down, one-up adaptive rule (i.e., the level decreased after a correct response and increased after an incorrect response). The SRT was thus defined as the SNR giving 50\% correct word recognition in the psychometric function (Levitt, 1971). The initial SNR was $10 \mathrm{~dB}$, and the speech level changed in $4 \mathrm{~dB}$ steps between words 1 and 14 , and in $2 \mathrm{~dB}$ steps between words 14 and 35. The SRT was calculated as the mean of the SNRs for the final 20 words. Feedback was not given to the participants on the correctness of their responses.

SRTs were measured in eight conditions: four without and four with compression. The conditions without compression included one without precursor, one with a steady precursor, one with a mildly fluctuating precursor, and one with a highly fluctuating precursor (the same no-precursor condition was taken as the reference to calculate the amount of adaptation). The four conditions with compression involved one without precursor and one with a highly fluctuating precursor times two compression ratios (2:1 and 4:1 dB/dB). Conditions without and with compression were administered in two separate blocks. The four conditions within each block were administered in random

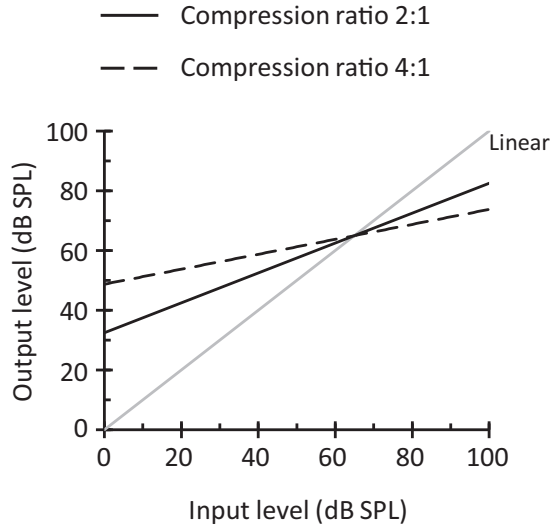

Figure 3. Compressor input/output functions for the two compression ratios used in the study.

order. Blocks with and without compression were alternated, and participants received a brief break after each block.

Three SRTs were obtained for each of the eight conditions, and their mean was taken as the SRT for that condition. An SRT measurement was discarded, and a new measurement was obtained when the SD within the measure was $>4 \mathrm{~dB}$. A fourth SRT was sometimes obtained and averaged when the experimenter deemed that the first three SRT measurements were too variable (no objective criterion was applied). To train subjects in the task with vocoded words, four SRTs were measured at the beginning of the experiment (conditions chosen at random) and discarded from the analyses.

During the measurements, participants were seated in a double-wall, sound-attenuating booth, and the presentation of each word was controlled by the experimenter, who was sitting outside the booth without visual interaction with the participant. A sound cue ( $1 \mathrm{kHz}$ pure tone with $500 \mathrm{~ms}$ duration) was presented $500 \mathrm{~ms}$ before the stimulus onset to warn the listener about the stimulus presentation and to focus his/her attention on the speech recognition task.

Controlling for the effects of mean precursor level. The mean levels of steady and highly fluctuating precursors were different 
( 65 vs $\sim 69 \mathrm{~dB}$ SPL, respectively). This difference would make it hard to know whether any differences in adaptation for the steady and the highly fluctuating precursor were related to their different level distributions or their different mean level. To elucidate this issue, we performed a complementary control experiment on $14 \mathrm{NH}$ listeners (4 males, mean \pm SD age: $29.2 \pm 9.3$ years). Audiometric thresholds were $\leq 20 \mathrm{~dB}$ HL at octave frequencies between 0.25 and $8 \mathrm{kHz}$ in the test (left) ear of all but 2 participants. One participant had a $25 \mathrm{~dB}$ HL threshold at $0.25 \mathrm{kHz}$, and another had a $25 \mathrm{~dB}$ HL threshold at $8 \mathrm{kHz}$. Only 1 of these subjects had participated in the main experiment with vocoded words (described above).

The experiment was performed for vocoded words presented in the left ear. It included three conditions: one without precursor, one with steady precursor at $65 \mathrm{~dB}$ SPL, and one with steady precursor at $69 \mathrm{~dB}$ SPL (the same mean level as the highly fluctuating precursor in the main experiment). The level of noise presented simultaneously with the word was fixed at $65 \mathrm{~dB}$ SPL. No compression was applied. We hypothesized that similar adaptation would occur for steady precursors of 65 and $69 \mathrm{~dB}$ SPL. This would support that a difference in adaptation for the steady and the highly fluctuating precursor in the main experiment would be related to differences in the level distributions rather than to the difference $(\sim 4 \mathrm{~dB})$ in mean levels.

The measurement procedure was as for the main experiment.

Apparatus. Stimuli were digitally stored and presented through custom-made MATLAB software version 2017 (The MathWorks; RRID:SCR_001622). Stimuli were played via an RME Fireface UCX soundcard at a sampling rate of $44.1 \mathrm{kHz}$ with 24 bit resolution. Stimuli were presented to the listeners using ER2 insert earphones (Etymotic Research), designed to give a flat frequency response at the eardrum from 250 to $8000 \mathrm{~Hz}$. Sound pressure levels were calibrated by placing the earphones in a Zwislocki DB-100 coupler connected to a sound level meter (Brüel Kjaer, mod. 2238). Calibration was performed at $1 \mathrm{kHz}$, and the obtained sensitivity was used at all other frequencies.

Statistical analyses. Statistical analyses were performed with SPSS Statistics, version 23 (IBM; RRID:SCR_002865). The Shapiro-Wilk test of normality revealed that the SRTs followed a Gaussian distribution for vocoded but not for natural words. For vocoded words, a one-way repeated-measures ANOVA was used to test for the effect of precursor type on SRTs for conditions without compression. For conditions with compression, paired Student's $t$ tests were used to compare mean SRTs with and without the highly fluctuating precursor. Separate tests were applied for each compression ratio. Friedman and Wilcoxon signed ranks tests (the nonparametric equivalents to repeated-measures ANOVA and Student's $t$ tests) were used to analyze SRTs for natural words. Differences in the amount of adaptation between natural and vocoded words and the possible interaction between word type and precursor type were tested with a two-way repeated-measures ANOVA (including word type and precursor type as factors).

To analyze the data of the control experiment aimed at investigating the effects of mean precursor level on noise adaptation, we performed a one-way repeated-measures ANOVA with the three conditions as a factor.

Post hoc pairwise comparisons (in the repeated-measures ANOVAs or the Friedman tests) were conducted using Bonferroni corrections for multiple comparisons. An effect was regarded as statistically significant when the null hypotheses could be rejected with $95 \%$ confidence (two-tailed, $p \leq 0.05$ ).

\section{CEOAEs}

Participants. We attempted to measure CEOAEs for 18 participants, but results from 7 participants were discarded because (1) CEOAE levels for 6 participants did not meet the SNR criterion (see below), and (2) the contralateral noise was erroneously presented at $\sim 55 \mathrm{~dB}$ SPL instead of $65 \mathrm{~dB}$ SPL for 1 participant. Therefore, reliable CEOAEs were measured for 11 participants, 2 of whom had participated in the main speech recognition tests. The mean \pm SD age of the participants was $30.5 \pm 9.9$ years. They had normal audiometric thresholds $(\leq 20 \mathrm{~dB}$ HL at octave frequencies between $125 \mathrm{~Hz}$ and $8 \mathrm{kHz}$ in both ears), except 1 participant whose audiometric thresholds were $25 \mathrm{~dB} \mathrm{HL}$ at 125 and $250 \mathrm{~Hz}$ in the left ear.

Stimuli. Clicks were presented to the left ear. CEOAEs were measured without contralateral acoustic stimulation (CAS), with a steady contralateral SSN, and with a highly fluctuating contralateral SSN. Clicks were presented at $60 \mathrm{~dB}$ SPL peak equivalent. The steady and highly fluctuating contralateral noises had mean levels of 65 and $\sim 69 \mathrm{~dB}$ SPL, respectively, the same levels as those used in the speech recognition task. Clicks were presented at a rate of $43.5 / \mathrm{s}$. The use of rates $\geq 31.25 / \mathrm{s}$ probably activates the ipsilateral MOCR (Boothalingam and Purcell, 2015). Hence, we probably assessed the effects of ipsilateral and contralateral MOCRs.

Procedure. Each measurement involved the presentation of 1000 stimulus trials. The nonlinear protocol was used (Kemp et al., 1990), and so each trial involved presenting three identical clicks of one polarity followed by a single click of three times the amplitude and opposite polarity. Twelve measures were obtained in total: four without CAS, four with contralateral steady noise, and four with contralateral highly fluctuating noise. Conditions were randomized. The mean CEOAE level across the four measures was taken as the CEOAE level for that condition. The recording probe remained in the participant's ear throughout the 12 measures to minimize the possible variance in the measure from altering the position of the probe in the ear canal. A measure was regarded as valid only when the CEOAE level was at least $6 \mathrm{~dB}$ above the noise floor (SNR $\geq 6 \mathrm{~dB}$ ).

Apparatus. Participants sat in a double-wall sound booth during testing. CEOAEs were measured with an Eclipse system (Interacoustics), suite version 1.0.1. The contralateral noise was digitally stored and presented through custom-made MATLAB software, played via an RME Fireface UCX soundcard at a sampling rate of $44.1 \mathrm{kHz}$ and with 24 bit resolution, and presented to the listeners using ER2 insert earphones.

Analyses. CEOAE amplitude was calculated over an analysis time window 4-20 ms after the click offset. The change in CEOAE amplitude, $\triangle \mathrm{CEOAE}$, was calculated as follows (Backus and Guinan, 2007; Mishra and Lutman, 2013; Bhatt, 2017; Boothalingam et al., 2019):

$$
\Delta \mathrm{CEOAE}[\%]=100 \times \frac{\mathrm{CEOAE}_{C T R}[\mathrm{~Pa}]-\mathrm{CEOAE}_{\text {CAS }}[\mathrm{Pa}]}{C E O A E_{C T R}[\mathrm{~Pa}]}
$$

where $\mathrm{CEOAE}_{\mathrm{CTR}}$ and $\mathrm{CEOAE}_{\mathrm{CAS}}$ denote CEOAE pressure amplitude in the control and CAS conditions, respectively, in units of Pascal.

The Shapiro-Wilk test of normality revealed that CEOAE values conformed to a Gaussian distribution. A one-way repeatedmeasures ANOVA with the three conditions (no CAS, steady CAS, highly fluctuating CAS) was used to test for the statistical significance of CAS on CEOAE amplitude. 
A

Natural words $(N=27)$

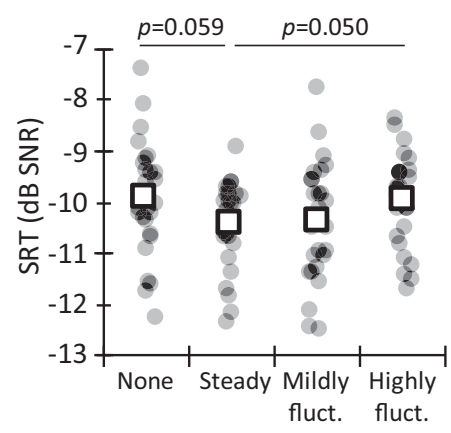

Precursor type

C Vocoded words $(N=15)$

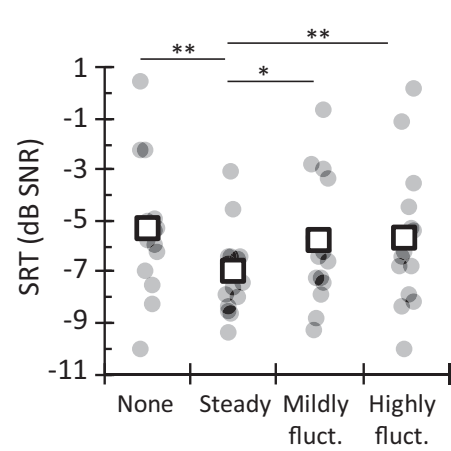

Precursor type
B Natural words $(N=27)$

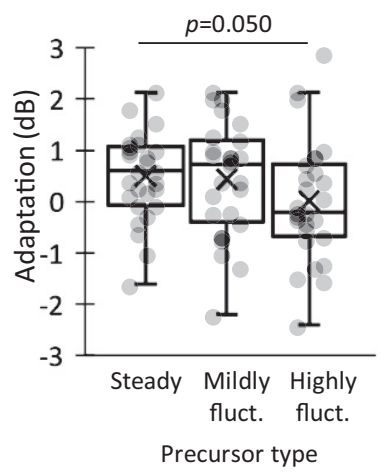

D Vocoded words $(N=15)$

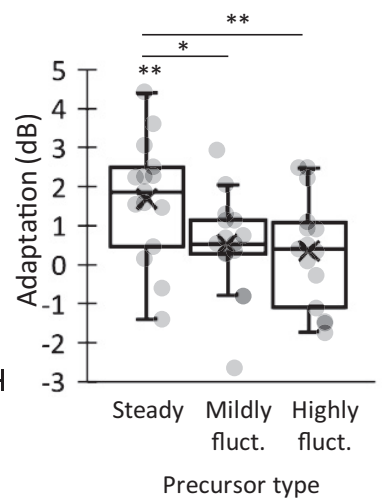

Figure 4. The effect of precursor-level statistics on SRTs and on noise adaptation. A, SRTs for natural words $(N=27)$ measured without precursor and with steady, mildly fluctuating, and highly fluctuating precursors. Dots and squares represent individual and mean scores, respectively. Each dot indicates the mean of at least three measures. $\boldsymbol{B}$, Adaptation to noise for natural words calculated as the difference between SRTs measured with and without precursor. Positive values indicate better (lower) SRTs in the condition with precursor than in the condition without precursor. Bottom, middle, and top lines in each box plot indicate the 25th, 50th (median), and 75th percentiles, respectively. Crosses represent mean values. Dots indicate individual results. $\boldsymbol{C}, \boldsymbol{D}$, The same as in $\boldsymbol{A}$ and $\boldsymbol{B}$, but for vocoded words $(N=15)$. ${ }^{*} p \leq 0.05,{ }^{* *} p \leq 0.01$.

\section{Results}

\section{Effects of precursor-level statistics on noise adaptation}

To investigate how the precursor-level distribution affected noise adaptation, we measured SRTs for noise-masked words presented without precursor as well as with precursors with different level statistics (steady, mildly fluctuating, and highly fluctuating). Figure $4 A$ shows SRTs for natural (unprocessed) words in these conditions. There was a significant effect of precursor type on SRTs (Friedman $\chi_{(3)}^{2}=9.8, N=27, p=0.020$ ). Post hoc pairwise comparisons revealed that SRTs tended to be better (lower) with the steady precursor than without precursor $(p=0.059)$. SRTs also tended to be better for the steady than for the highly fluctuating precursor $(p=0.050)$. The mean amount of adaptation was $0.50 \mathrm{~dB}( \pm 0.8 \mathrm{SD}), 0.44 \mathrm{~dB}( \pm 1.1 \mathrm{SD})$, and $0.02 \mathrm{~dB}( \pm 1.2 \mathrm{SD})$ for steady, mildly fluctuating, and highly fluctuating precursors, respectively (Fig. $4 B$ ). This indicates that greater fluctuations in the noise precursor generated less noise adaptation.

Figure $4 C$ shows SRTs for vocoded words for the same conditions as described above. There was a significant effect of precursor type on SRTs (repeated-measures ANOVA $F_{(3,42)}=8.2$, $N=15, p<0.001$ ). Post hoc pairwise comparisons revealed significantly better SRTs with the steady precursor than without

precursor $(p=0.005)$. SRTs were also better with the steady precursor than with the mildly fluctuating precursor $(p=0.037)$ or with the highly fluctuating precursor $(p=0.008)$. The mean amount of adaptation was $1.69 \mathrm{~dB}( \pm 1.5 \mathrm{SD}), 0.51 \mathrm{~dB}( \pm 1.3 \mathrm{SD})$, and $0.36 \mathrm{~dB}( \pm 1.4 \mathrm{SD})$ for steady, mildly fluctuating, and highly fluctuating precursors, respectively (Fig. $4 D$ ). In other words, as it occurred for natural words, noise adaptation was greatest for the constant-level precursor.

One might think that the envelope fluctuations in the highly fluctuating precursor could mask (or interfere with) those of the words, and thus hinder word recognition in a way similar to how an amplitude modulated (AM) precursor hinders the detection of an AM probe when both have the same modulation rate (Oxenham and Dau, 2001; Wojtczak and Viemeister, 2005; Wojtczak et al., 2011). This, however, did not seem to be the case because mean SRTs with the highly fluctuating precursor were not statistically different from SRTs without precursor (Fig. 4). In other words, the highly fluctuating precursor did not hinder word recognition and thus cannot be regarded as an "interferer."

\section{Effects of instantaneous amplitude compression on noise adaptation}

To investigate the effects of instantaneous amplitude compression on noise adaptation, we measured SRTs for noise-masked words presented with and without a highly fluctuating precursor. Stimuli were passed through a broadband, instantaneous amplitude compressor before presenting them to the listeners. Because instantaneous compression reduces precursor-level fluctuations (Fig. 2E,F), it was expected to facilitate noise adaptation. Figure $5 A$ shows SRTs for natural words for the two precursor conditions (none and highly fluctuating) and for the two compression ratios tested (2:1 and 4:1). For the compression ratio 2:1, SRTs were not significantly different with and without precursor (Wilcoxon test $Z=-1.29 ; p=0.196$ ). For the compression ratio 4:1, however, SRTs were better with than without precursor (Wilcoxon test $Z=-2.2 ; p=0.028$ ). Adaptation was, on average, $0.25 \mathrm{~dB}( \pm 1.0 \mathrm{SD})$ and $0.55 \mathrm{~dB}( \pm 1.2 \mathrm{SD})$ for the compression ratios $2: 1$ and $4: 1$, respectively (Fig. $5 B$ ).

Figure $5 C$ shows corresponding SRTs for vocoded words. SRTs were better with than without precursor for the compression ratio of 2:1 (paired Student's $t$ test: $t_{(14)}=5.92 ; p<0.001$ ) as well as for the compression ratio of 4:1 $\left(t_{(14)}=3.56 ; p=0.003\right)$. The mean adaptation was $2.16 \mathrm{~dB}( \pm 1.4 \mathrm{SD})$ and $2.47 \mathrm{~dB}( \pm 2.7$ $\mathrm{SD})$ for the compression ratios $2: 1$ and 4:1, respectively, and it was not significantly different for the two compression ratios $\left(t_{(14)}=-0.44 ; p=0.664\right)$.

In addition to the findings just reported, SRTs were overall worse with than without compression (compare Fig. $4 A$ vs Fig. $5 A$ or Fig. $4 C$ vs Fig. $5 C$ ). In other words, compression deteriorated intelligibility as expected.

\section{Comparison of noise adaptation for natural and vocoded words}

Figure $6 \mathrm{~A}$ compares noise adaptation for natural and vocoded words across all tested conditions. Only data for the 14 participants tested with natural and vocoded words were analyzed to make an intrasubject comparison. Adaptation was overall greater for vocoded than for natural words (repeated-measures ANOVA: $\left.F_{(1,13)}=16.5, p=0.001\right)$. The interaction between word type and test condition was also significant $\left(F_{(4,52)}=3.5, p=0.013\right)$. Post hoc analyses revealed that adaptation was greater for vocoded than for natural words in the conditions with the steady precursor $(p=0.023)$, with the highly fluctuating precursor compressed with a ratio 2:1 


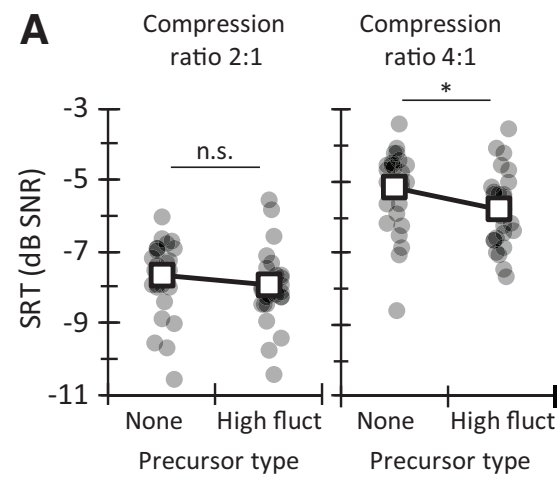

B
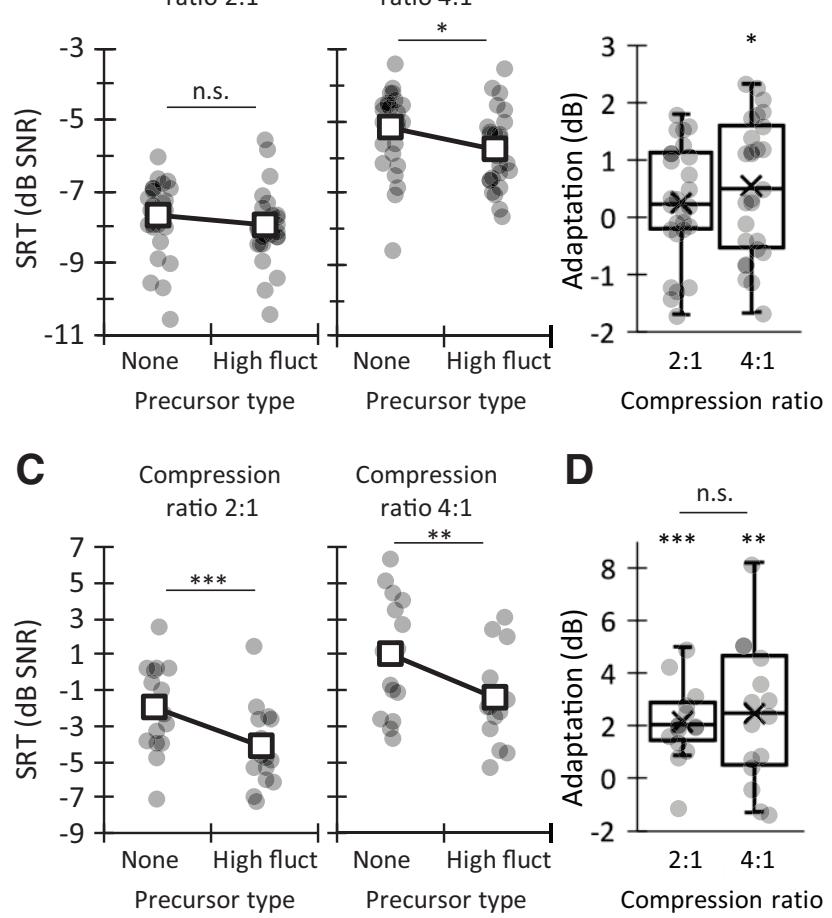

Figure 5. The effect of fast-amplitude compression on SRTs and on noise adaptation. $\boldsymbol{A}$, SRTs for natural words $(N=27)$ measured without precursor and with a highly fluctuating precursor. Both words and noise were compressed with compression ratios of 2:1 or 4:1, as indicated at the top. Dots and squares represent individual and mean scores, respectively. $\boldsymbol{B}$, Adaptation to noise for natural words calculated as the difference between SRTs with and without precursor. Positive values indicate better (lower) SRTs in the condition with precursor. $\boldsymbol{C}, \boldsymbol{D}$, SRTs and adaptation for vocoded words $(N=15)$. The layout is as for $\boldsymbol{A}$ and $\boldsymbol{B}$. ${ }^{*} p \leq 0.05,{ }^{* *} p \leq 0.01,{ }^{* * *} p \leq 0.001$. n.s.: not significant.

A

$\square$ Natural words

Vocoded words

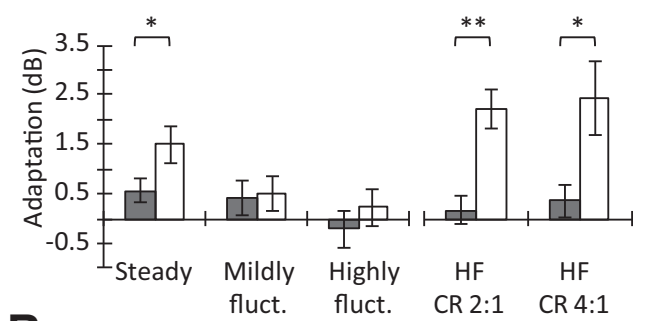

B

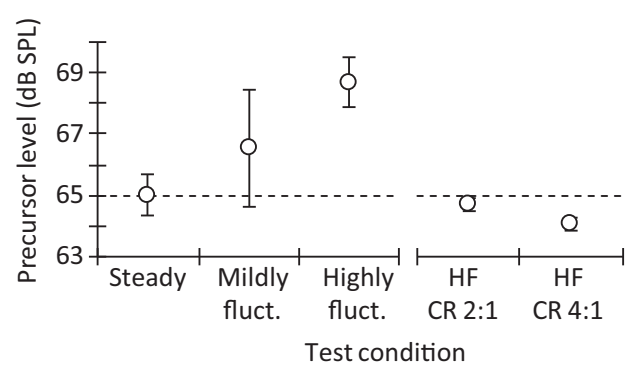

Figure 6. Mean adaptation for natural and vocoded words $(\boldsymbol{A})$ and mean precursor level (B) for all tested conditions. $A$, Group mean scores for those participants tested with natural and vocoded words $(N=14)$. Error bars indicate SEM. ${ }^{*} p \leq 0.05,{ }^{* *} p \leq 0.01$. $B$, Mean precursor level for each condition. Error bars indicates SD. HF, Highly fluctuating; $C R$, Compression ratio.

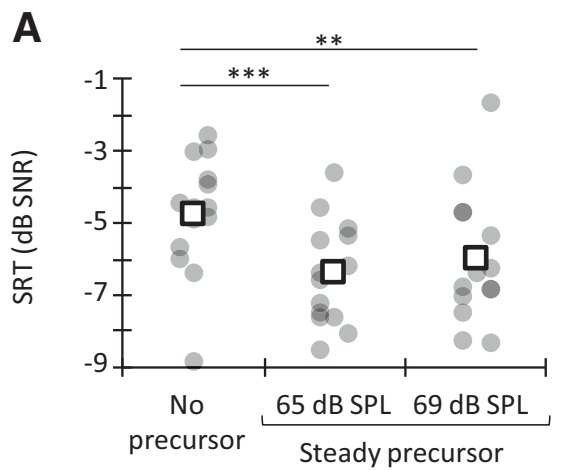

B

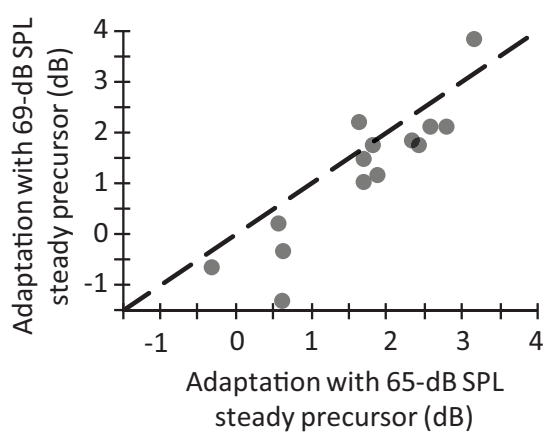

Figure 7. Effects of precursor mean level on SRTs $(\boldsymbol{A})$ and on noise adaptation $(\boldsymbol{B})$ for vocoded words. $\boldsymbol{A}$, Individual (dots) and mean (squares) SRTs. ${ }^{* *} p \leq 0.01,{ }^{* * *} p \leq 0.001$. $\boldsymbol{B}$, Comparison of adaptation for steady precursors at 65 and $69 \mathrm{~dB}$ SPL. Points below the dashed line indicate greater adaptation for the 65 than for the $69 \mathrm{~dB}$ SPL precursor.

$(p=0.004)$, and with the highly fluctuating precursor compressed with a ratio 4:1 $(p=0.012)$. The greater adaptation for vocoded than for natural words was consistent with the findings of our previous study (Marrufo-Pérez et al., 2018).

\section{The effect of mean precursor level on noise adaptation}

When stimuli were not compressed, participants showed adaptation when the precursor was steady but not when it was fluctuating (Fig. 6A). It is uncertain whether this was because of the difference in level fluctuations or because of the difference in mean level across precursor types (Fig. 6B). To elucidate the reason, we measured adaptation for vocoded words for a steady precursor at $69 \mathrm{~dB}$ SPL, the same level as that of the highly fluctuating precursor in the main experiment. For completeness, we also measured adaptation for a steady precursor level at $65 \mathrm{~dB}$ SPL, the same level as that of the simultaneous masking noise.

Figure 7 shows the individual and mean SRTs. Analyses revealed better SRTs with than without precursor [repeatedmeasures ANOVA: $\left.F_{(2,26)}=19.5, p<0.001\right]$. Post hoc analyses revealed that SRTs were better with the steady precursor of $65 \mathrm{~dB}$ SPL than without precursor $(p<0.001)$. SRTs were also better with the steady precursor of $69 \mathrm{~dB}$ SPL than without precursor $(p=0.012)$. Figure $7 B$ shows adaptation for each participant. Most subjects showed slightly greater adaptation with the $65 \mathrm{~dB}$ SPL precursor than with the $69 \mathrm{~dB}$ SPL steady precursor (1.65 vs $1.27 \mathrm{~dB}$ ), but the difference was not statistically significant $(p=0.129)$.

In summary, there was adaptation for steady precursors but not for highly fluctuating precursors with equal mean levels (69 dB SPL). This shows that precursor-level distribution is important for noise adaptation. In addition, the slightly greater adaptation with the $65 \mathrm{~dB}$ SPL precursor than with the $69 \mathrm{~dB}$ SPL 

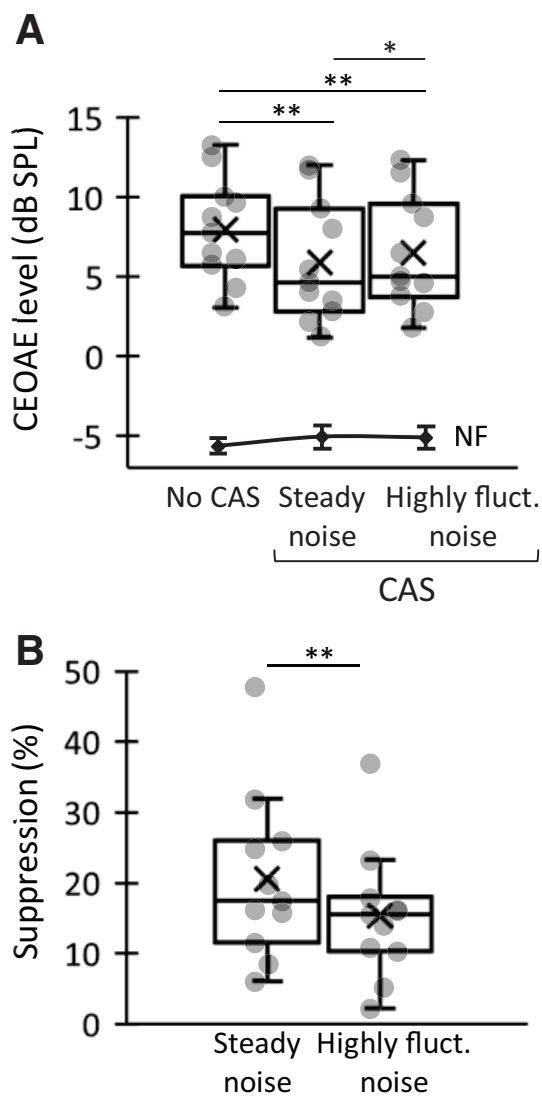

Figure 8. The effect of CAS on CEOAE levels. $A$, CEOAE levels for 11 participants measured without CAS, with contralateral steady SSN, and with contralateral highly fluctuating SSN. $\mathrm{NF}$, Noise floor (mean $=-5.5 \mathrm{~dB}$ SPL). Error bars indicate $1 \mathrm{SD} . \boldsymbol{B}$, CEOAE suppression calculated using Equation 2. ${ }^{*} p \leq 0.05,{ }^{* *} p \leq 0.01$.

steady precursor demonstrated that the mean precursor level (relative to the simultaneous noise) may be important for noise adaptation.

CEOAE suppression by steady and highly fluctuating noise To investigate the potential role of the MOCR in noise adaptation, we measured the suppression of CEOAEs by contralateral steady and highly fluctuating noises. The effect of contralateral steady and highly fluctuating noises on CEOAE levels is depicted in Figure $8 \mathrm{~A}$. There was a significant effect of CAS on CEOAE levels [repeated-measures ANOVA: $F_{(2,20)}=22.6$, $p<0.001$. Post hoc pairwise comparisons revealed that both the steady noise and the highly fluctuating noise suppressed CEOAEs ( $p=0.002$ for the two noise types), but suppression was greater for the steady than for the fluctuating noise $(2.1 \mathrm{~dB}$ vs $1.5 \mathrm{~dB} ; p=0.016)$. This difference in suppression $(0.6 \mathrm{~dB})$ is similar to that observed by Mertes (2018) $(0.5 \mathrm{~dB})$ after using $60 \mathrm{~dB}$ SPL contralateral broadband noises with and without being modulated with the envelope of a four-talker babble. The latter modulation resembles the modulation in the present highly fluctuating noise (see Fig. 1 in Mertes, 2018). Figure $8 B$ shows the amount of suppression calculated using Equation 2.

\section{Discussion}

We have shown that, for NH listeners, the recognition of noisemasked words improves when words are preceded by steady (constant level) noise but not when they are preceded by highly fluctuating noise with the same mean level (Figs. 4, 7). In addition, we have shown that instantaneous amplitude compression facilitates adaptation in highly fluctuating noise (Fig. 5) because it reduces the variability in noise level, effectively making a highly fluctuating noise more stationary. Together, the present results indicate that the variability in precursor level is important for noise adaptation to occur, and are consistent with neural DRA toward the most common level in the environment as being the underlying mechanism.

\section{The role of the medial olivocochlear reflex in noise adaptation}

MOCR activation can theoretically facilitate speech in noise recognition by (1) enhancing auditory nerve responses to sounds embedded in noise (Nieder and Nieder, 1970a,b; Kawase et al., 1993), (2) restoring the discharge rate range of auditory nerve fibers in noise to values observed in quiet (Winslow and Sachs, 1988), and/or (3) adapting the neuronal dynamic range to the sound-level statistics (Dean et al., 2005, 2008). We have shown that a highly fluctuating noise significantly suppressed CEOAEs when presented contralaterally to the test ear but did not improve word recognition when it preceded noise-masked words ipsilaterally. Because ipsilateral broadband noise is as effective as (if not more than) contralateral broadband noise in activating the MOCR (Lilaonitkul and Guinan, 2009), this suggests that the highly fluctuating noise precursor probably activated the MOCR during the present speech tests, and yet it did not produce noise adaptation. In other words, this suggests that the MOCR played little or no role in noise adaptation. This conclusion is supported by two other studies. Wojtczak et al. (2019) showed that AM sensitivity in noise can improve with a precursor even when the precursor does not change basilar membrane responses (i.e., when it does not activate the MOCR). Second, cochlear-implant users show adaptation to noise in word recognition despite lacking MOCR effects (Marrufo-Pérez et al., 2018), which suggests that mechanisms different from the MOCR can facilitate speech-innoise recognition, at least when the MOCR is absent.

\section{Instantaneous compression facilitates adaptation to noise}

We have shown that applying broadband, fast-amplitude compression to the stimuli facilitates noise adaptation. Compression occurs at multiple stages in the auditory system. For example, the velocity of motion of the basilar membrane grows compressively with increasing sound level (Robles and Ruggero, 2001), and the inner hair cell (IHC) applies additional compression (Lopez-Poveda and Eustaquio-Martín, 2006) such that the IHC receptor potential grows with increasing sound level more compressively than does basilar membrane motion (Patuzzi and Sellick, 1983). Cochlear mechanical compression (Vavakou et al., 2019) and IHC compression (e.g., Dallos, 1986) are both fast enough that they could reduce the level fluctuations in the background noise, thereby making the IHC representation of a fluctuating sound less fluctuating than the corresponding acoustic input. Therefore, it is tempting to hypothesize that auditory compression facilitates noise adaptation. The data from Dubno et al. (2012) appear to be consistent with this idea. They did not measure noise adaptation but showed that the recognition of vocoded speech-in-noise at a constant SNR was better at midlevels (60 dB SPL), where basilar membrane responses are more compressive, than at lower or higher speech levels, where basilar membrane responses are more linear. They also reported that subjects with more compressive basilar membrane responses showed greater changes in recognition scores as speech level 
increased. The better recognition scores at mid-levels may have occurred because basilar membrane compression reduced the noise level fluctuations more at mid-levels, and thus induced greater neural DRA to the noise statistics and better speech-innoise recognition.

Hearing-impaired (HI) listeners show less or no noise adaptation in word recognition (at least for babble maskers) (BenDavid et al., 2012) or AM detection (Jennings et al., 2018). Because hearing loss is typically associated with combined loss or dysfunction of cochlear IHCs and OHCs (Liberman and Dodds, 1984; Lopez-Poveda and Johannesen, 2012; Johannesen et al., 2014), and the MOCR is thought to have antimasking effects (for review, see Guinan, 2006; Lopez-Poveda, 2018), the conventional view is that $\mathrm{OHC}$ loss would impair noise adaptation because $\mathrm{OHCs}$ are necessary for the MOCR to exert its antimasking effects (Jennings et al., 2018; for review, see Lopez-Poveda, 2018). If this were the case, however, it would be hard to explain why HI listeners show less or no noise adaptation while cochlear-implant users show normal adaptation even though the two listener groups are expected to have impaired or absent MOCR effects (Marrufo-Pérez et al., 2018, 2019). The present findings prompt us to hypothesize a different explanation: listeners with hearing loss (OHC loss) may show less noise adaptation not because they have an impaired MOCR but because they have less or no auditory peripheral compression (Plack et al., 2004; LopezPoveda et al., 2005; Johannesen et al., 2014). Reduced or absent compression would make the IHC receptor potential representation more fluctuating than normal, and neurons would not have a prevailing level to adapt to. Cochlear implants, however, include instantaneous compression at the back end of processing to map the wide range of acoustic pressure into a narrower range of electrical current (e.g., Wilson et al., 1991; Lopez-Poveda et al., 2016). This compression reduces the level fluctuations of incoming sounds and probably facilitates neuronal DRA toward a prevailing stimulus level.

Further research is necessary to investigate the potential role of basilar membrane, IHC, or other forms of auditory compression, as well compression in hearing devices, in noise adaptation.

Noise adaptation was less for natural than for vocoded words Like Marrufo-Pérez et al. (2018), here we also found less noise adaptation for natural than for vocoded words (Fig. 6). MarrufoPérez et al. (2018) explained this difference by ceiling effects; that is, they reasoned that adaptation is overall less for conditions where baseline SRTs (without precursor) are better. Consistent this explanation, here, baseline SRTs were better for natural than for vocoded words. It is uncertain, however, whether other factors can explain or contribute to the reduced adaptation for natural words. For instance, NH listeners use TFS cues in steady noise backgrounds when those cues are available (Hopkins and Moore, 2009) and adaptation mechanisms seem to enhance only envelope cues (Marrufo-Pérez et al., 2018). It is possible that envelope enhancement is less useful when speech recognition relies to some extent in a nonenhanced (e.g., TFS) cue.

\section{Implications}

The present findings have several implications. First, we have provided new evidence that noise adaptation is unlikely mediated by the MOCR. This new evidence adds to other recent evidence supporting the same conclusion (Marrufo-Pérez et al., 2018; Wojtczak et al., 2019) and contributes to resolving the long-standing controversy about the role of the MOCR in facilitating speech-in-noise recognition (for review, see LopezPoveda, 2018).

Second, we have demonstrated that instantaneous amplitude compression facilitates noise adaptation. This could be relevant for hearing aid design. For instance, there is no consensus on whether hearing aid release times should be a few or several hundreds of milliseconds (e.g., Jenstad and Souza, 2005; Kowalewski et al., 2018). In fluctuating noise, adaptation would be expected to occur for fast but not for slow release times because the hearing aid would work like a linear amplifier and would not reduce the level fluctuations in the noise in the latter case. Of course, the broadband, fast compressor used here would be inadequate because it distorts the speech, and the negative effects of this distortion outweigh the positive effects of adaptation (i.e., the present SRTs with a highly fluctuating precursor were still overall better without than with compression; Figs. 4, 5). However, it may be possible to design narrowband compressors that are fast enough to facilitate noise adaptation with minimal distortion (e. g., compressors based on basilar-membrane models) (e.g., Lopez-Poveda and Meddis, 2001; Clark et al., 2018).

Third, the lesser noise adaptation for natural than for vocoded words suggests that adaptation may be greater for those listeners who rely on speech envelope cues, such as cochlearimplant users (Shannon et al., 1995; Schatzer et al., 2010), or for those listeners with degraded ability to use TFS cues, such as older listeners with clinically NH (Füllgrabe, 2013). It also suggests that $\mathrm{HI}$ listeners might have significant trouble understanding audible speech in noise because, on the one hand, they are less able to use TFS speech cues for speech recognition (Buss et al., 2004; Lorenzi et al., 2006), which makes them more reliant on envelope cues, and on the other hand, they do not benefit from noise adaptation because they show much less or no peripheral compression if their hearing loss involves $\mathrm{OHC}$ loss or dysfunction (Plack et al., 2004; Lopez-Poveda et al., 2005; Johannesen et al., 2014).

\section{References}

Ainsworth WA, Meyer GF (1994) Recognition of plosive syllables in noise: comparison of an auditory model with human performance. J Acoust Soc Am 96:687-694.

American National Standards Institute (1996) S3.6 Specification for audiometers. New York: American National Standards Institute.

Bacon SP, Fay RR, Popper AN (2004) Compression: from cochlea to cochlear implants. New York: Springer.

Backus BC, Guinan JJ (2007) Measurement of the distribution of medial olivocochlear acoustic reflex strengths across normal-hearing individuals via otoacoustic emissions. J Assoc Res Otolaryngol 8:484-496.

Bakay WM, Anderson LA, Garcia-Lazaro JA, McAlpine D, Schaette R (2018) Hidden hearing loss selectively impairs neural adaptation to loud sound environments. Nat Commun 9:4298.

Ben-David BM, Tse VY, Schneider BA (2012) Does it take older adults longer than younger adults to perceptually segregate a speech target from a background masker? Hear Res 290:55-63.

Berlin CI, Hood LJ, Wen H, Szabo P, Cecola RP, Rigby P, Jackson DF (1993) Contralateral suppression of non-linear click-evoked otoacoustic emissions. Hear Res 71:1-11.

Bhatt I (2017) Increased medial olivocochlear reflex strength in normal-hearing, noise-exposed humans. PLoS One 12:e0184036.

Boothalingam S, Allan C, Allen P, Purcell DW (2019) The medial olivocochlear reflex is unlikely to play a role in listening difficulties in children. Trends Hear 23:1-25.

Boothalingam S, Purcell DW (2015) Influence of the stimulus presentation rate on medial olivocochlear system assays. J Acoust Soc Am 137:724732.

Buss E, Hall JW, Grose JH (2004) Temporal fine-structure cues to speech and pure tone modulation in observers with sensorineural hearing loss. Ear Hear 25:242-250. 
Cárdenas MR, Marrero V (1994) Cuaderno de logoaudiometría. Madrid: Universidad Nacional de Educación a Distancia.

Cervera T, Gonzalez-Alvarez J (2007) Temporal effects of preceding bandpass and band-stop noise on the recognition of voiced stops. Acta Acust United Acust 93:1036-1045.

Clark NR, Lecluyse W, Jürgens T (2018) Analysis of compressive properties of the BioAid hearing aid algorithm. Int J Audiol 57:S130-S138.

Cooper NP, Guinan JJ (2003) Separate mechanical processes underlie fast and slow effects of medial olivocochlear efferent activity. J Physiol 548:307-312.

Cooper NP, Guinan JJ (2006) Efferent-mediated control of basilar membrane motion. J Physiol 576:49-54.

Collet L, Kemp DT, Veuillet E, Duclaux R, Moulin A, Morgon A (1990) Effect of contralateral auditory stimuli on active cochlear micro-mechanical properties in human subjects. Hear Res 43:251-262.

Costalupes JA, Young ED, Gibson DJ (1984) Effects of continuous noise backgrounds on rate response of auditory nerve fibers in cat. J Neurophysiol 51:1326-1344.

Dallos P (1986) Neurobiology of cochlear inner and outer hair cells: intracellular recordings. Hear Res 22:185-198.

Dean I, Harper NS, McAlpine D (2005) Neural population coding of sound level adapts to stimulus statistics. Nat Neurosci 8:1684-1689.

Dean I, Robinson BL, Harper NS, McAlpine D (2008) Rapid neural adaptation to sound-level statistics. J Neurosci 28:6430-6438.

Dolan DF, Guo MH, Nuttall AL (1997) Frequency-dependent enhancement of bas-ilar membrane vibration during olivocochlear bundle stimulation. J Acoust Soc Am 102:3587-3596.

Dubno JR, Ahlstrom JB, Wang X, Horwitz AR (2012) Level-dependent changes in perception of speech envelope cues. J Assoc Res Otolaryngol 13:835-852.

Evans EF (1974) Auditory frequency selectivity and the cochlear nerve. In: Facts and models in hearing. (Zwicker E, Terhardt E, eds). SpingerVerlag: Berlin-Heidelberg.

Füllgrabe C (2013) Age-dependent changes in temporal-fine-structure processing in the absence of peripheral hearing loss. Am J Audiol 22:313-315.

Garcia-Lazaro JA, Ho SS, Nair A, Schnupp JW (2007) Shifting and scaling adaptation to dynamic stimuli in somatosensory cortex. Eur J Neurosci 26:2359-2368

Gibson DJ, Young ED, Costalupes JA (1985) Similarity of dynamic range adjustment in auditory nerve and cochlear nuclei. J Neurophysiol 53: 940-958.

Glasberg B, Moore BC (1989) Psychoacoustic abilities of subjects with unilateral and bilateral cochlear impairments and their relationship to the ability to understand speech. Scand Audiol Suppl 32:1-25.

Guinan JJ (2006) Olivocochlear efferents: anatomy, physiology, function, and the measurement of efferent effects in humans. Ear Hear 27:589-607.

Herrmann B, Maess B, Johnsrude IS (2018) Aging affects adaptation to sound-level statistics in human auditory cortex. J Neurosci 38:1989-1999.

Herrmann B, Augereau T, Johnsrude IS (2019) Neural responses and perceptual sensitivity to sound depend on sound-level statistics. Sci Rep 10:9571.

Higgins ET, Semin GR (2001) Communication and social psychology. In: International encyclopedia of social and behavioral sciences. Oxford: Elsevier Science.

Hopkins K, Moore BC (2009) The contribution of temporal fine structure to the intelligibility of speech in steady and modulated noise. J Acoust Soc Am 125:442-446.

Jennings SG, Chen J, Fultz SE, Ahlstrom JB, Dubno JR (2018) Amplitude modulation detection with a short-duration carrier: effects of a precursor and hearing loss. J Acoust Soc Am 143:2232-2243.

Jenstad LM, Souza PE (2005) Quantifying the effect of compression hearing aid release time on speech acoustics and intelligibility. J Speech Lang Hear Res 48:651-667.

Johannesen PT, Pérez-González P, Lopez-Poveda EA (2014) Across-frequency behavioral estimates of the contribution of inner and outer hair cell dysfunction to individualized audiometric loss. Front Neurosci 8:214.

Johannesen PT, Pérez-González P, Kalluri S, Blanco JL, Lopez-Poveda EA (2016) The influence of cochlear mechanical dysfunction, temporal processing deficits, and age on the intelligibility of audible speech in noise for hearing-impaired listeners. Trends Hear 7:20.
Kawase T, Delgutte B, Liberman MC (1993) Antimasking effects of the olivocochlear reflex: II. Enhancement of auditory-nerve response to masked tones. J Neurophysiol 70:2533-2549.

Kemp DT, Ryan S, Bray P (1990) A guide to the effective use of otoacoustic emissions. Ear Hear 11:93-105.

Kowalewski B, Zaar J, Fereczkowski M, MacDonald EN, Strelcyk O, May T, Dau T (2018) Effects of slow- and fast-acting compression on hearingimpaired listeners' consonant-vowel identification in interrupted noise. Trends Hear 22:1-12.

Levitt H (1971) Transformed up-down methods in psychoacoustics. J Acoust Soc Am 49:467-677.

Lilaonitkul W, Guinan JJ (2009) Human medial olivocochlear reflex: effects as functions of contralateral, ipsilateral, and bilateral elicitor bandwidths. J Assoc Res Otolaryngol 10:459-470.

Liberman MC, Dodds LW (1984) Single-neuron labeling and chronic cochlear pathology: III. Stereocilia damage and alterations of threshold tuning curves. Hear Res 16:55-74.

Lopez-Poveda EA (2018) Olivocochlear efferents in animals and humans: from anatomy to clinical relevance. Front Neurol 9:197.

Lopez-Poveda EA, Eustaquio-Martín A (2006) A biophysical model of the inner hair cell: the contribution of potassium currents to peripheral auditory compression. J Assoc Res Otolaryngol 7:218-235.

Lopez-Poveda EA, Johannesen PT (2012) Behavioral estimates of the contribution of inner and outer hair cell dysfunction to individualized audiometric loss. J Assoc Res Otolaryngol 13:485-504.

Lopez-Poveda EA, Meddis R (2001) A human nonlinear cochlear filterbank. J Acoust Soc Am 110:3107-3118.

Lopez-Poveda EA, Plack CJ, Meddis R, Blanco JL (2005) Cochlear nonlinearity in listeners with moderate sensorineural hearing loss. Hear Res 205:172-183.

Lopez-Poveda EA, Eustaquio-Martín A, Stohl JS, Wolford RD, Schatzer R, Wilson BS (2016) A binaural cochlear implant sound coding strategy inspired by the contralateral medial olivocochlear reflex. Ear Hear 37:138-148.

Lorenzi C, Gilbert G, Carn H, Garnier S, Moore BC (2006) Speech perception problems of the hearing impaired reflect inability to use temporal fine structure. Proc Natl Acad Sci USA 103:18866-18869.

Marrufo-Pérez MI, Eustaquio-Martín A, Lopez-Poveda EA (2018) Adaptation to noise in human speech recognition unrelated to the medial olivocochlear reflex. J Neurosci 38:4138-4145.

Marrufo-Pérez MI, Eustaquio-Martín A, Fumero MJ, Gorospe JM, Polo R, Gutiérrez-Revilla A, Lopez-Poveda EA (2019) Adaptation to noise in amplitude modulation detection without the medial olivocochlear reflex. Hear Res 377:133-141.

Mertes IB (2018) Human medial efferent activity elicited by dynamic versus static contralateral noises. Hear Res 365:100-109.

Mishra SK, Lutman ME (2013) Repeatability of click-evoked otoacoustic emission-based medial olivocochlear efferent assay. Ear Hear 34:789798.

Murugasu E, Russell IJ (1996) The effect of efferent stimulation on basilar membrane displacement in the basal turn of the guinea pig cochlea. J Neurosci 16:325-332.

Nieder P, Nieder I (1970a) Stimulation of efferent olivocochlear bundle causes release from low level masking. Nature 227:184- 185.

Nieder P, Nieder I (1970b) Antimasking effect of crossed olivocochlear bundle stimulation with loud clicks in guinea pig. Exp Neurol 28:179-188.

Ohzawa I, Sclar G, Freeman RD (1982) Contrast gain control in the cat visual cortex. Nature 298:266-268.

Oxenham AJ, Dau T (2001) Modulation detection interference: effects of concurrent and sequential streaming. J Acoust Soc Am 110:402-408.

Patuzzi R, Sellick PM (1983) A comparison between basilar membrane and inner hair cell receptor potential input-output functions in the guinea pig cochlea. J Acoust Soc Am 74:1734-1741.

Phillips DP, Hall SE (1986) Spike-rate intensity functions of cat cortical neurons studied with combined tone-noise stimuli. J Acoust Soc Am 80:177187.

Plack CJ, Drga V, Lopez-Poveda EA (2004) Inferred basilar-membrane response functions for listeners with mild to moderate sensorineural hearing loss. J Acoust Soc Am 115:1684-1695.

Rees A, Palmer AR (1989) Neuronal responses to amplitude-modulated and pure-tone stimuli in the guinea pig inferior colliculus, and their modification by broadband noise. J Acoust Soc Am 85:1978-1994. 
Robinson BL, Harper NS, McAlpine D (2016) Meta-adaptation in the auditory midbrain under cortical influence. Nature Comm 7:13442.

Robles L, Ruggero MA (2001) Mechanics of the mammalian cochlea. Physiol Rev 81:1305-1352.

Rocchi F, Ramachandran R (2018) Neuronal adaptation to sound statistics in the inferior colliculus of behaving macaques does not reduce the effectiveness of the masking noise. J Neurophysiol 120:2819-2833.

Rosen S (1992) Temporal information in speech: acoustic, auditory and linguistic aspects. Philos Trans R Soc Lond B Biol Sci 336:367-373.

Schatzer R, Krenmayr A, Au DK, Kals M, Zierhofer C (2010) Temporal fine structure in cochlear implants: preliminary speech perception results in Cantonese-speaking implant users. Acta Otolaryngol 130:1031-1039.

Shannon RV, Zeng FG, Kamath V, Wygonski J, Ekelid M (1995) Speech recognition with primarily temporal cues. Science 270:303-304.

Vavakou A, Cooper NP, van der Heijden M (2019) The frequency limit of outer hair cell motility measured in vivo. eLife $8: 1-18$.

Watkins PV, Barbour DL (2008) Specialized neuronal adaptation for preserving input sensitivity. Nat Neurosci 11:1259-1261.

Watkins PV, Barbour DL (2011) Rate-level responses in awake marmoset auditory cortex. Hear Res 275:30-42.
Wen B, Wang GI, Dean I, Delgutte B (2009) Dynamic range adaptation to sound-level statistics in the auditory nerve. J Neurosci 29:13797-13808.

Wen B, Wang GI, Dean I, Delgutte B (2012) Time course of dynamic range adaptation in the auditory nerve. J Neurophysiol 108:69-82.

Wilson BS, Finley CC, Lawson DT, Wolford R, Eddington DK, Rabinowitz WM (1991) Better speech recognition with cochlear implants. Nature 352:236-238.

Winslow RL, Sachs MB (1988) Single-tone intensity discrimination based on auditory-nerve rate responses in backgrounds of quiet, noise, and with stimulation of the crossed olivocochlear bundle. Hear Res 35:165-190.

Wojtczak M, Viemeister NF (2005) Forward masking of amplitude modulation: basic characteristics. J Acoust Soc Am 118:3198-3210.

Wojtczak M, Nelson PC, Viemeister NF, Carney LH (2011) Forward masking in the amplitude-modulation domain for tone carriers: psychophysical results and physiological correlates. J Assoc Res Otolaryngol 12:361373.

Wojtczak M, Klang AM, Torunsky NT (2019) Exploring the role of medial olivocochlear efferents on the detection of amplitude modulation for tones presented in noise. J Assoc Res Otolaryngol 20:395-413. 